\title{
Calculated Coupling Efficiency Between an Elliptical-Core Optical Fiber and an Optical Waveguide Over Temperature
}

\author{
Margaret L. Tuma, ${ }^{1}$ Andreas Weisshaar, ${ }^{2}$ Jian Li, ${ }^{2}$ and Glenn Beheim ${ }^{1}$ \\ ${ }^{1}$ NASA Lewis Research Center \\ Cleveland, OH 44135 \\ ${ }^{2}$ Oregon State University, Department of Electrical and Computer Engineering \\ Corvallis, OR 97331
}

\begin{abstract}
To determine the feasibility of coupling the output of a single-mode optical fiber into a single-mode rib waveguide in a temperature varying environment, a theoretical calculation of the coupling efficiency between the two was investigated. Due to the complex geometry of the rib guide, there is no analytical solution to the wave equation for the guided modes, thus, approximation and/or numerical techniques must be utilized to determine the field patterns of the guide. In this study, three solution methods were used for both the fiber and rib guide fields; the effective-index method (EIM), Marcatili's approximation, and a Fourier method. These methods were utilized independently to calculate the electric field profile of each component at two temperatures, $20^{\circ} \mathrm{C}$ and $300^{\circ} \mathrm{C}$, representing a nominal and high temperature.
\end{abstract}

Using the electric field profile calculated from each method, the theoretical coupling efficiency between an ellipticalcore optical fiber and a rib waveguide was calculated using the overlap integral and the results were compared. It was determined that a high coupling efficiency can be achieved when the two components are aligned. The coupling efficiency was more sensitive to alignment offsets in the $y$ direction than the $x$, due to the elliptical modal field profile of both components. Changes in the coupling efficiency over temperature were found to be minimal.

Keywords: coupling efficiency, rib waveguide, elliptical fiber, Effective-index method, Marcatili's method, Fourier method

\section{INTRODUCTION}

To model the behavior of an optical device over temperature, changes in both the refractive index and physical dimensions of the layers must be determined. Temperature-induced changes in the materials due to thermal expansion and stress are taken into account to determine device properties at elevated temperatures.

This research explored the coupling efficiency between an elliptical-core optical fiber and a rib waveguide. The optical fiber ${ }^{1}$ transmits the source light into the guide, which serves as the input to an integrated optic pressure sensor. Because this sensor is to be used in a temperature varying environment on an airplane, changes in the coupling efficiency over temperature are of interest. The sensor design and fabrication were performed at the University of 


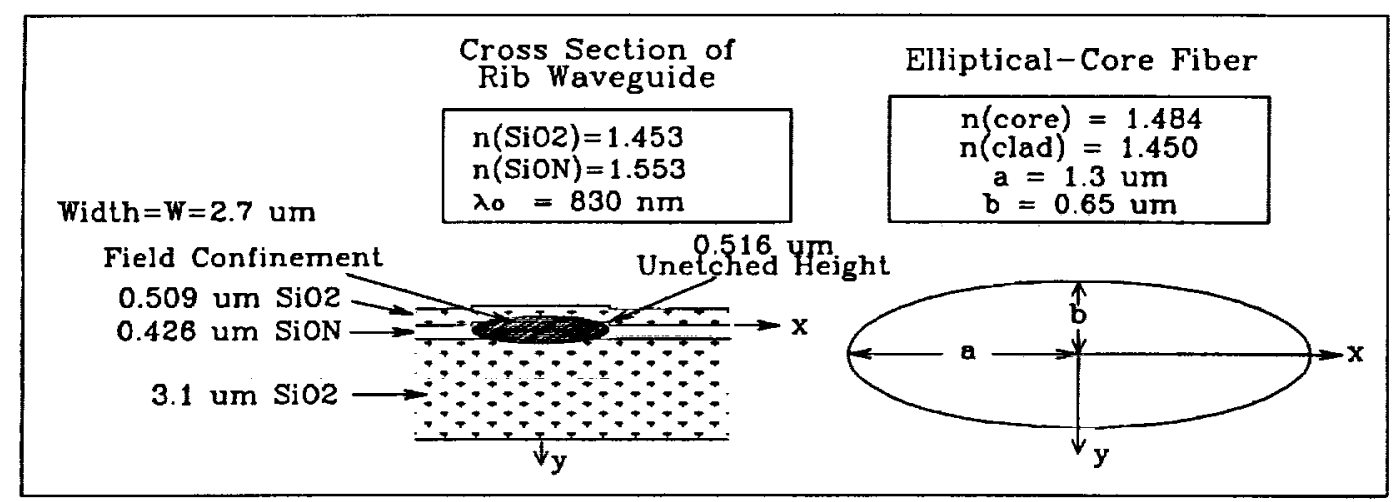

Figure 1: Cross sections of rib waveguide and elliptical-core optical fiber.

Cincinnati. ${ }^{2}$ Both the rib and fiber physical parameters are illustrated in Figure 1.

\section{FIELD SOLUTION METHODS}

There is no exact solution for the field profile of a rib guide, however, there is an analytical solution for the fiber but it cannot be solved in closed form. ${ }^{3}$ It was determined that the three techniques used to determine the rib field were also applicable to the fiber. ${ }^{4-5}$ The effective-index method (EIM) and Marcatili's method were chosen for the approximation techniques, and the Fourier method was chosen for the numerical technique, and was expected to produce the most accurate field results. All three methods provide solutions to the scalar wave equation,

$$
\frac{\partial^{2} E_{x}(x, y)}{\partial x^{2}}+\frac{\partial^{2} E_{x}(x, y)}{\partial y^{2}}+\left[n^{2}(x, y) k_{o}^{2}-\beta^{2}\right] E_{x}(x, y)=0
$$

where $E_{x}(x, y)$ is the field profile, $n(x, y)$ is the spatially dependent function for the index of refraction, $\beta$ is the propagation constant, and $k_{o}$ equals $\frac{2 \pi}{\lambda_{o}}$ where $\lambda_{o}$ is the free space wavelength, $830 \mathrm{~nm}$. The scalar-wave approximation is valid if the difference between the guide core and cladding index is small,

$$
\Delta=\frac{n_{1}-n_{2}}{n_{1}} \ll<1
$$

where $n_{1}$ is the core and $n_{2}$ is the cladding index. ${ }^{6}$ For the rib waveguide $\Delta=0.064$ and for the fiber $\Delta=0.023$, satisfying the weakly guiding condition. For quasi-TE modes propagating in the $z$ direction, the electric field is of the form

$$
\vec{E}(x, y, z, t)=\hat{x} E_{x}(x, y) e^{i(\omega t-\beta z)} .
$$

\subsection{Effective-Index Method (EIM)}

\subsubsection{Rib Waveguide - EIM}

The $\mathrm{EIM}^{7-11}$ reduces the two-dimensional scalar wave equation, equation (1), into two one-dimensional problems. This is achieved by dividing the rib guide into separate planar guides as described in a previous paper. ${ }^{12}$ Mathematically, the EIM employs separation of variables to solve the scalar-wave equation, where the index profile is 
approximated as

$$
n^{2}(x, y)=n^{\prime 2}(x)+n^{\prime \prime 2}(y) .
$$

In equation (4), the quantity $n^{\prime \prime}(y)$ is denoted as

$$
\begin{array}{ll}
n^{\prime \prime 2}(y)=n_{1}^{2} & \text { for } y<\left|b^{\prime}\right| \\
n^{\prime \prime 2}(y)=n_{2}^{2} & \text { for } y<-b^{\prime} \\
n^{\prime \prime 2}(y)=n_{3}^{2} & \text { for } y>b^{\prime}
\end{array}
$$

and $n^{\prime 2}(x)$ as

$$
\begin{array}{ll}
n^{\prime 2}(x)=0 & \text { for }|x|<a^{\prime} \\
n^{\prime 2}(x)=-\left[\frac{\beta_{y}^{2}}{k_{o}^{2}}-n_{4}^{2}\right] & \text { for }|x|>a^{\prime}
\end{array}
$$

where $\beta_{y}$ is the propagation constant solution for the $y$ dependent field. Substituting the results for $n^{\prime 2}(x)$ and $n^{\prime \prime 2}(y)$ into equation (4) yields the separable refractive index profile, $n^{2}(x, y)$, for which the EIM solves exactly. ${ }^{13}$ The electric field profile of the rib is the product of the field distribution of the two one-dimensional planar slabs, $X(x)$ and $Y(y)$,
expressed as

$$
E_{x}(x, y)=X(x) Y(y)
$$

\subsubsection{Fiber - EIM}

The fiber shown in Figure 1 is a single-mode polarization-maintaining elliptical-core fiber. This fiber was approximated as a rectangle with dimensions $a^{\prime}$ and $b^{\prime}$ such that the rectangle and ellipse have the same area and aspect ratio, given by ${ }^{14}$

$$
a^{\prime}=a \frac{\sqrt{\pi}}{2} \quad \text { and } \quad b^{\prime}=b \frac{\sqrt{\pi}}{2} .
$$

Using the procedure given in section 2.1.1, the fiber field was determined for this rectangle.

\subsection{Marcatili's Method}

\subsubsection{Rib Wavegulde - Marcatill's Method}

For modes far from cutoff, the majority of the field energy is confined to the central core region. If this assumption applies, a pseudo-rectangular waveguide can be constructed to approximate the rib structure. ${ }^{15-17}$ The cross section of the waveguide is divided into nine regions as shown in Figure 2, where $t$ is the etch depth, $r$ is the height of the etched $\mathrm{SiON}$ layer, such that the unetched height is $r+t$.

Analogous to the EIM, this method reduces the two-dimensional wave equation into two separate one-dimensional problems as described in a previous publication. ${ }^{12}$ The pseudo-guide 4 is assigned a refractive index profile which approximates that of the actual rib and is defined by ${ }^{17}$

$$
n^{2}(x, y)=n^{\prime 2}(x)+n^{\prime 2}(y)-n_{1}^{2}
$$

where

$$
\begin{array}{ll}
n^{\prime}(x)=n_{1} & \text { for }|x|<W / 2 \\
n^{\prime}(x)=n_{2} & \text { for }|x|>W / 2
\end{array}
$$




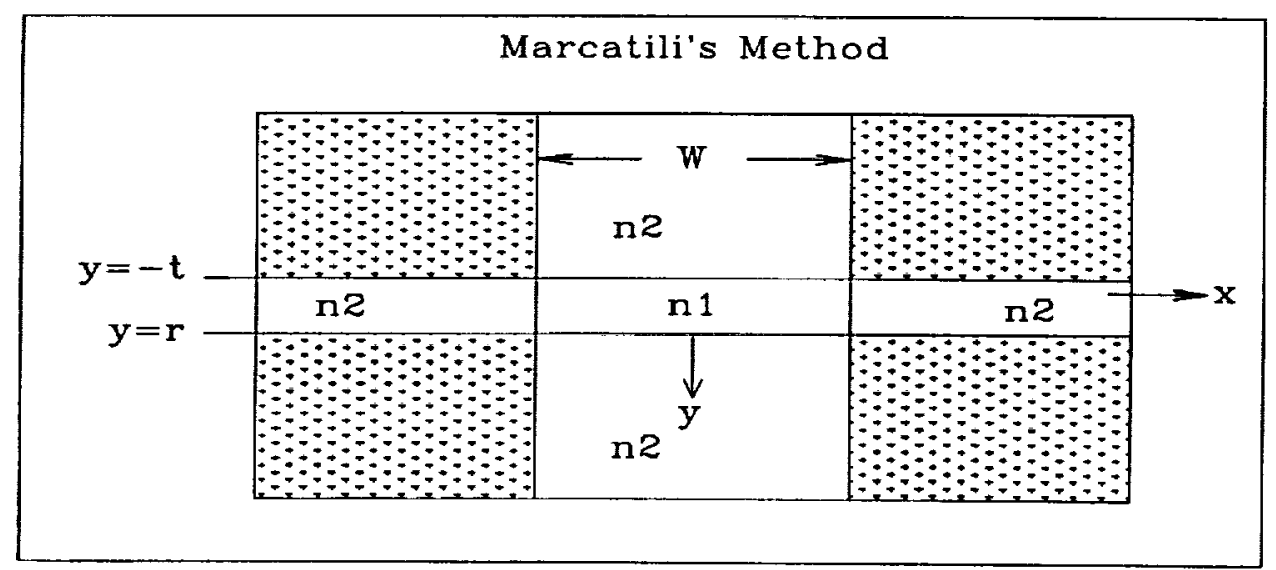

Figure 2: Marcatili's method: pseudo-guide used to approximate rib guide.

and

$$
\begin{array}{ll}
n^{\prime \prime}(y)=n_{1} & \text { for }-t<y<r \\
n^{\prime \prime}(y)=n_{2} & \text { for }(y<-t) \text { or }(y>r)
\end{array}
$$

\subsubsection{Fiber - Marcatill's Method}

Marcatili's technique can be used to calculate the field profile of an elliptical-core fiber. ${ }^{4,16}$ The elliptical core is approximated by a rectangle, for which an analytical solution can be found. The same rectangle dimensions chosen for the EIM are used here. ${ }^{4}$ Using this rectangle as the pseudo-guide Marcatili's method, as described in section 2.2.1, is used to determine the fiber field profile.

\subsection{Fourier Method}

\subsubsection{RIb Waveguide - Fourier Method}

For the numerical technique, a solution to the scalar wave equation is obtained by expressing $E_{x}(x, y)$ as an infinite series in terms of a complete set of orthonormal functions. ${ }^{18-19}$ That is,

$$
E_{x}(x, y)=\sum_{\mu=1}^{\infty} \sum_{\nu=1}^{\infty} A_{\mu \nu} \Psi_{\mu \nu}(x, y)
$$

where

$$
\Psi_{\mu \nu}(x, y)=\frac{1}{\sqrt{L_{x} L_{y}}} \sin \left(\sigma_{\mu} x\right) \sin \left(\rho_{\nu} y\right),
$$

is a simple and complete orthonormal set of sine functions, and

$$
\sigma_{\mu}=\frac{\mu \pi}{L_{x}} \text { and } \rho_{\nu}=\frac{\nu \pi}{L_{y}}
$$


where $\mu$ and $\nu$ are integers. The sine function, $\Psi_{\mu \nu}$, is orthogonal over the finite base domain, $\left(0 \leq x \leq L_{x}\right)$ and $\left(0 \leq y \leq L_{y}\right)$, such that

$$
\int_{0}^{L_{\Sigma}} \int_{0}^{L_{y}} \Psi_{\mu \nu}(x, y) \Psi_{\mu^{\prime} \nu^{\prime}}(x, y) d x d y=\delta_{\mu \mu^{\prime}} \delta_{\nu \nu^{\prime}}
$$

For this method, the field is only defined over this finite base domain; thus, proper choice of the domain limits is critical for accurate results. The values for $L_{x}$ and $L_{y}$ are chosen such that the field on those boundaries is zero. For well-guided modes these boundaries are small and this method performs well, however, for waves at or near cutoff this method does not apply.

To obtain a matrix equation, equation (17) is inserted into the scalar wave equation, equation (1). Then, the left hand side is multiplied by $\Psi_{\mu \nu}(x, y)$ and integrated over the finite base domain producing

$$
\sum_{\mu=1}^{m_{x}} \sum_{\nu=1}^{m_{\nu}}\left(M_{\mu^{\prime} \nu^{\prime}, \mu \nu} A_{\mu \nu}\right)=\left(\frac{\beta^{2}}{k_{0}^{2}}\right) A_{\mu^{\prime} \nu^{\prime}}
$$

where

$$
M_{\mu^{\prime} \nu^{\prime}, \mu \nu}=\frac{4}{L_{x} L_{\nu}} \int_{0}^{L_{x}} \int_{0}^{L_{\nu}}\left[n^{2}(x, y)-\frac{\sigma_{\mu}^{2}-\rho_{\nu}^{2}}{k_{o}^{2}}\right] \Psi_{\mu \nu}(x, y) \Psi_{\mu^{\prime} \nu^{\prime}}(x, y) d x d y .
$$

In order to obtain a closed form solution for the integral, the index of refraction profile in equation (22) is approximated by a set of rectangles, each having a constant refractive index. ${ }^{19}$

Using the procedure from Marcuse, ${ }^{18}$ two FORTRAN programs ${ }^{20}$ were developed to determine the propagation characteristics of the rib and fiber. The $L_{x}$ and $L_{y}$ chosen values, $15 \mu \mathrm{m}$ and $10 \mu \mathrm{m}$, respectively, were large compared to the fiber and rib guide geometry, but necessary to maintain the zero field condition on the domain boundary while the rib guide was shifted with respect to the fiber. This was done to determine the coupling efficiency at various transverse offsets. Increasing the number of terms, $m_{x}$ and $m_{y}$, improves the accuracy until a convergence point is reached. Beyond that point, further increases in the number of expansion terms have negligible effect. It was determined that a series of 30 sine functions in both directions was sufficient to produce accurate results.

\subsubsection{Fiber - Fourier Method}

The Fourier method was applied to the elliptical-core fiber using the development in section 2.3.1 to calculate the field profile. The fiber core was approximated as a single rectangle with the integration taken over the elliptical-core boundary. The same $L_{x}$ and $L_{y}$ domain boundaries and expansion terms used for the rib were used for the fiber.

\section{Coupling Efficiency Calculation}

There are several causes of interconnect loss between a fiber and a waveguide. ${ }^{21-23}$ These include mode field mismatch, transverse offset ( $x, y$ offset), longitudinal separation ( $z$ offset), angular offset, and refractive-index mismatch. The loss mechanisms addressed in this study were the transverse offset and mode field mismatch. Mode field mismatch contributes significantly to interconnect loss, ${ }^{23-24}$ hence modeling is a necessary tool to design a waveguide whose field profile closely matches that of the input fiber. As misalignment in the $x$ and $y$ directions is inevitable in practice, the theoretical coupling efficiency results for $x$ and $y$ offsets are of interest.

Once the electric fields of the rib guide and fiber were calculated, the coupling efficiency between the two compo- 
nents, $\eta$, is calculated using the overlap integral, ${ }^{25}$

$$
\eta=\frac{\left|\iint E_{f}(x, y) E_{g}^{*}(x, y) d x d y\right|^{2}}{\iint E_{f}(x, y) E_{f}^{*}(x, y) d x d y \iint E_{g}(x, y) E_{g}^{*}(x, y) d x d y}
$$

where the integrals are taken over all space and $E_{f}(x, y)$ and $E_{g}(x, y)$ are the electric field profiles of the fiber and rib waveguide, respectively. For the EIM and Marcatili's method, this integration is performed directly. For the Fourier method, the coupling efficiency is determined as the integral of the product of the fiber and rib guide fields squared, denoted

$$
\left(\sum_{\mu} \sum_{\nu} A_{\mu \nu} B_{\mu \nu}\right)^{2}
$$

$A_{\mu \nu}$ and $B_{\mu \nu}$ are the coefficients of the fiber and rib guide, given in equation (17), which are normalized as

$$
\sum_{\mu} \sum_{\nu} A_{\mu \nu}^{2}=\sum_{\mu} \sum_{\nu} B_{\mu \nu}^{2}=1
$$

The major axis of the elliptical-core fiber was oriented parallel to the $x$ axis of the guide and the position of zero $x$ and $y$ offset was chosen to correspond to alignment of the maximum field positions of the fiber and rib. ${ }^{28}$ After solving for the aligned case, various $x$ offsets were introduced and a new coupling efficiency calculated for each offset at both temperatures. This procedure was then repeated for various $y$ offsets while the $x$ offset was fixed at zero.

\section{RESULTS}

\subsection{Field Results}

Theoretical field results for the rib using the three methods are illustrated in Figures 3 - 5 with the rib cross-section superimposed. For all the contour plots in this paper, each contour indicates a region of equal field magnitude in increments of 0.1 , with the maximum field magnitude being equal to 1.0 .

Field results using the EIM were very similar to those using the Fourier method, yet required considerably less computation time. The difference between the fields calculated using the EIM and Fourier method is shown in Figure 6. Marcatili's method assumes a tightly confined field, hence field results were tightly confined. The difference between the field calculated using Marcatili's method and the Fourier method is illustrated in Figure 7.

Modeling the elliptical fiber as a rectangle, field results using the EIM and Marcatili's method are shown in Figures 8-9, with the fiber cross-section superimposed. Field results from the Fourier numerical technique are shown in Figure 10. Differences between the electric field profile calculated using the EIM and Marcatili's method with respect to the Fourier method are shown in Figures 11 and 12. From these graphs, it appears that the field calculated using the EIM more closely matches that using the Fourier method than the field calculated using Marcatili's method. 


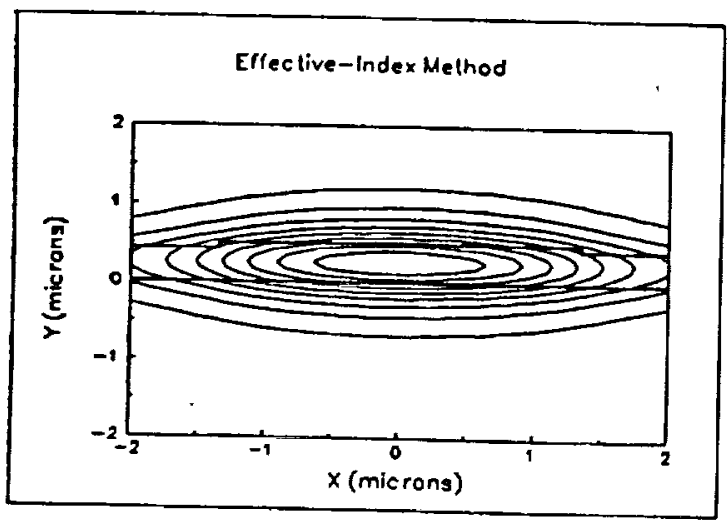

Figure 3: EIM: rib field contours.

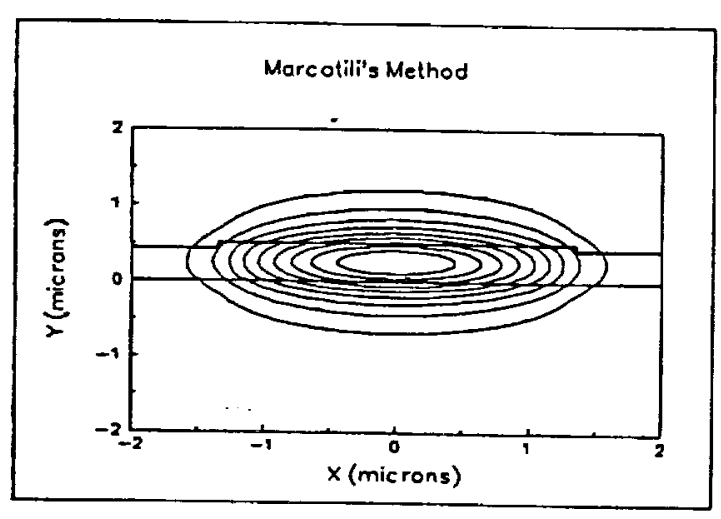

Figure 4: Marcatili's method: rib field contours.

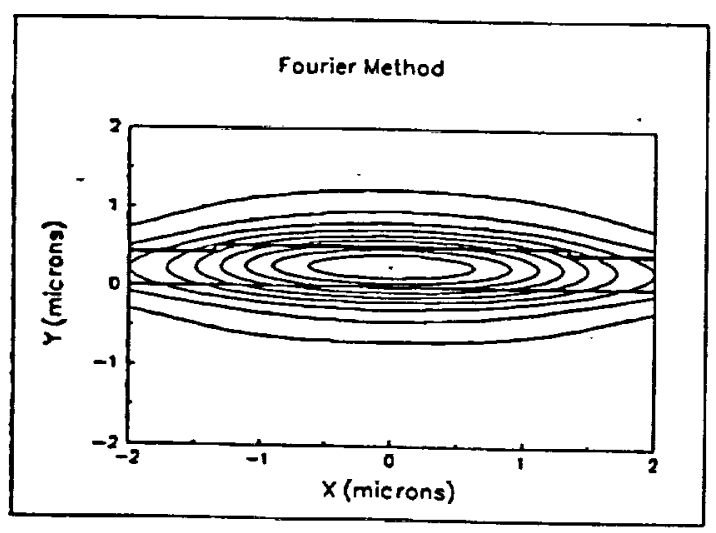

Figure 5: Fourier Method: rib field contours.

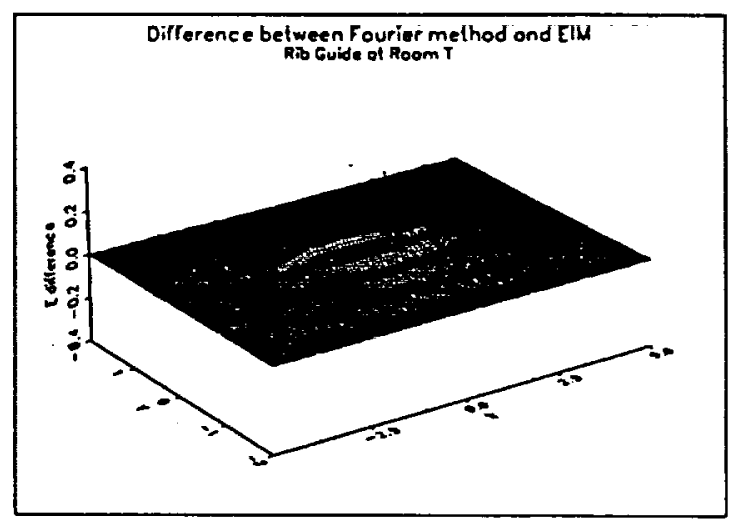

Figure 6: Difference - Fourier and EIM fields.

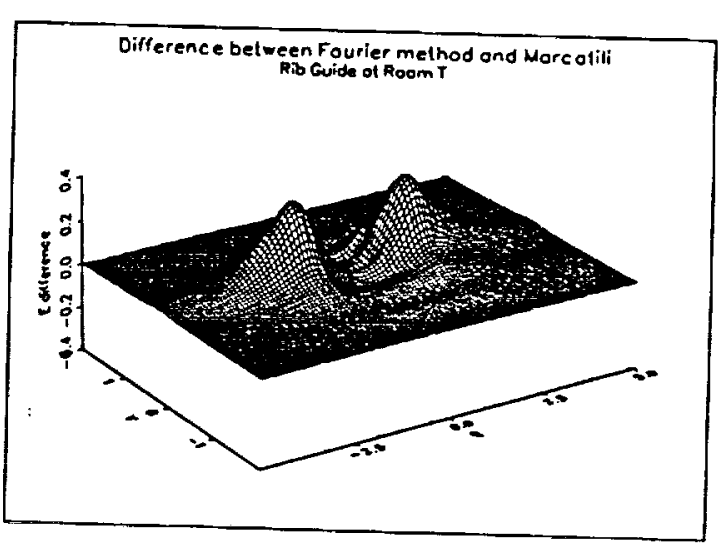

Figure 7: Difference - Fourier and Marcatili fields. 


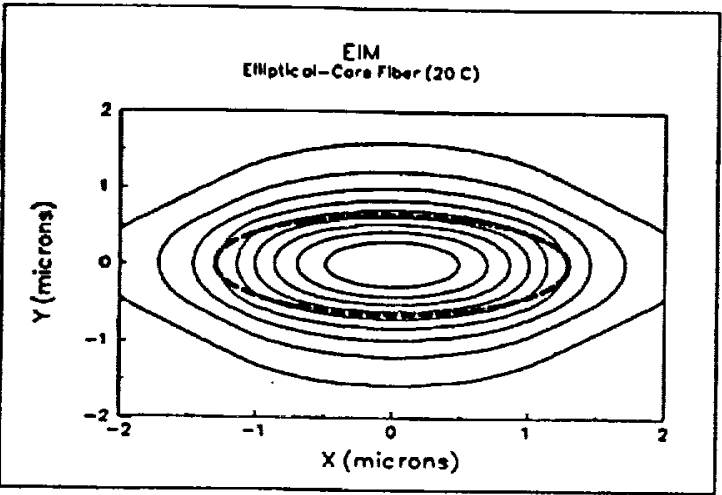

Figure 8: EIM: elliptical fiber field contours.

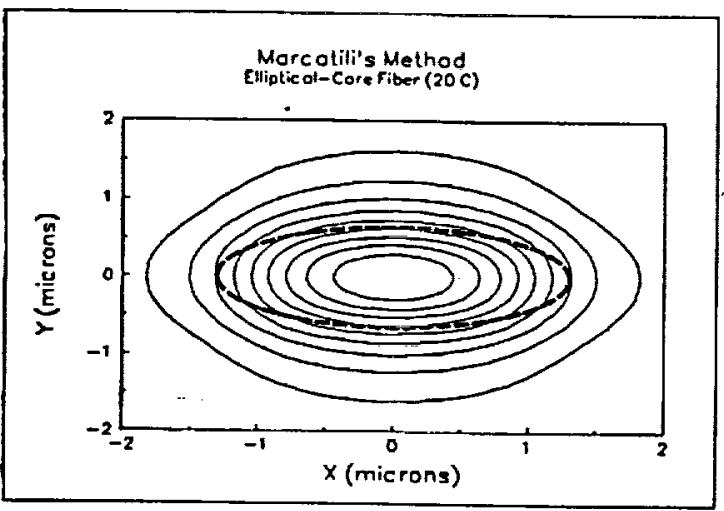

Figure 9: Marcatili's method: elliptical fiber field contours. Figure 12: Difference - Fourier and Marcatili fields.

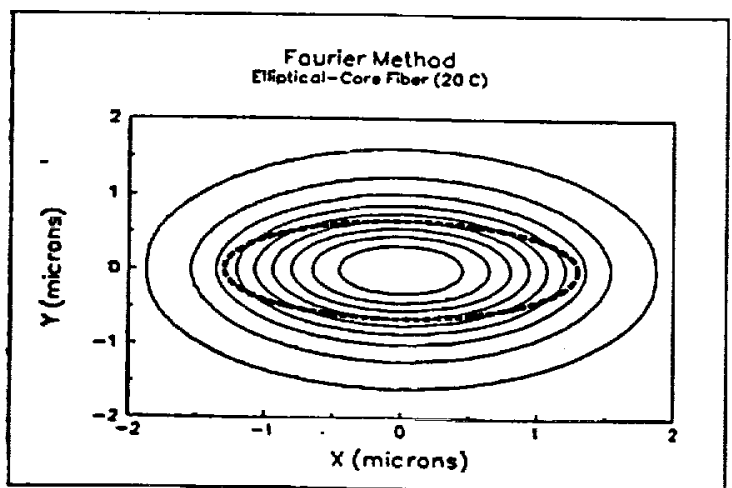

Figure 10: Fourier method: elliptical fiber field contours.
Figure 11: Difference - Fourier and EIM fields.

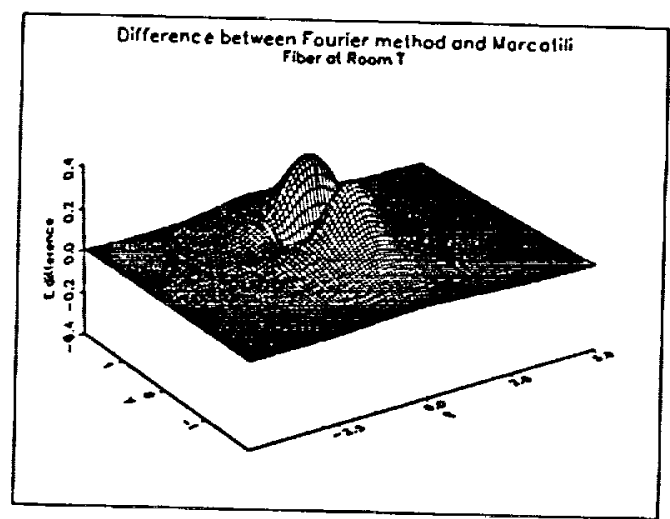

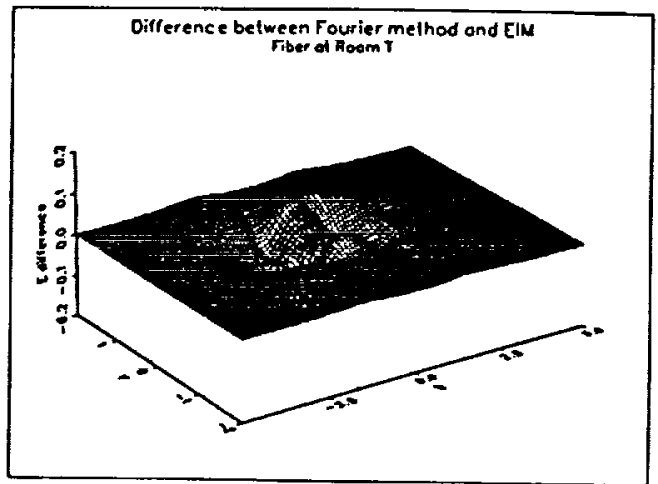




\subsection{Physical Changes Due to Temperature - Results}

Compared to the $20^{\circ} \mathrm{C}$ data, the rib and fiber parameters changed slightly when calculated at $300^{\circ} \mathrm{C}$, taking into account thermal expansion and stress with the results given in Table 1. It was apparent that the substrate stretched the film causing the rib width to increase while its height decreased. The indices of the rib and fiber all increased with increasing temperature. Temperature induced change in the rib indices was two orders of magnitude greater

Field contours for both the rib and fiber at $300^{\circ}$ were nearly identical to those calculated at $20^{\circ} \mathrm{C}$, using each of the solution methods, but are not shown due to space constraints.

\begin{tabular}{|l|l|l|}
\hline PARAMETER & $20^{\circ} \mathrm{C}$ & $300^{\circ} \mathrm{C}$ \\
\hline$W\left(300^{\circ} \mathrm{C}\right)$ & $2.700 \mu \mathrm{m}$ & $2.702 \mu \mathrm{m}$ \\
\hline$t_{\text {overlayer }}\left(300^{\circ} \mathrm{C}\right)$ & $0.509 \mu \mathrm{m}$ & $0.509 \mu \mathrm{m}$ \\
\hline$t_{\text {unetched }}\left(300^{\circ} \mathrm{C}\right)$ & $0.516 \mu \mathrm{m}$ & $0.516 \mu \mathrm{m}$ \\
\hline$t_{\text {etched }}\left(300^{\circ} \mathrm{C}\right)$ & $0.426 \mu \mathrm{m}$ & $0.426 \mu \mathrm{m}$ \\
\hline$n_{\text {overlayer }}\left(300^{\circ} \mathrm{C}\right)$ & 1.453 & 1.456 \\
\hline$n_{\text {rib }}\left(300^{\circ} \mathrm{C}\right)$ & 1.553 & 1.556 \\
\hline$n_{\text {isolation }}\left(300^{\circ} \mathrm{C}\right)$ & 1.453 & 1.456 \\
\hline
\end{tabular}

Table 1: Rib Guide Parameter Changes With Temperature

\begin{tabular}{|l|l|l|}
\hline PARAMETER & $20^{\circ} \mathrm{C}$ & $300^{\circ} \mathrm{C}$ \\
\hline$a$ & $1.300 \mu \mathrm{m}$ & $1.302 \mu \mathrm{m}$ \\
\hline$b$ & $0.650 \mu \mathrm{m}$ & $0.651 \mu \mathrm{m}$ \\
\hline$n_{\text {core }}$ & 1.484 & 1.487 \\
\hline$n_{\text {cladding }}$ & 1.450 & 1.453 \\
\hline
\end{tabular}

Table 2: Fiber Parameter Change With Temperature 


\subsection{Coupling Efficiency Results}

The $x$ direction offset ranged from $-3 \mu m$ to $+3 \mu m$ in $0.10 \mu m$ increments. Coupling efficiency results between the fiber and rib guide, calculated using the three different methods, are shown in Figure 13 as a function of this $x$ offset. It was evident from this graph that the EIM and the Fourier method predict similar results. Marcatili's method predicts the fields of both components to be more tightly confined in the $x$ direction, producing a coupling efficiency more sensitive to an $x$ offset.

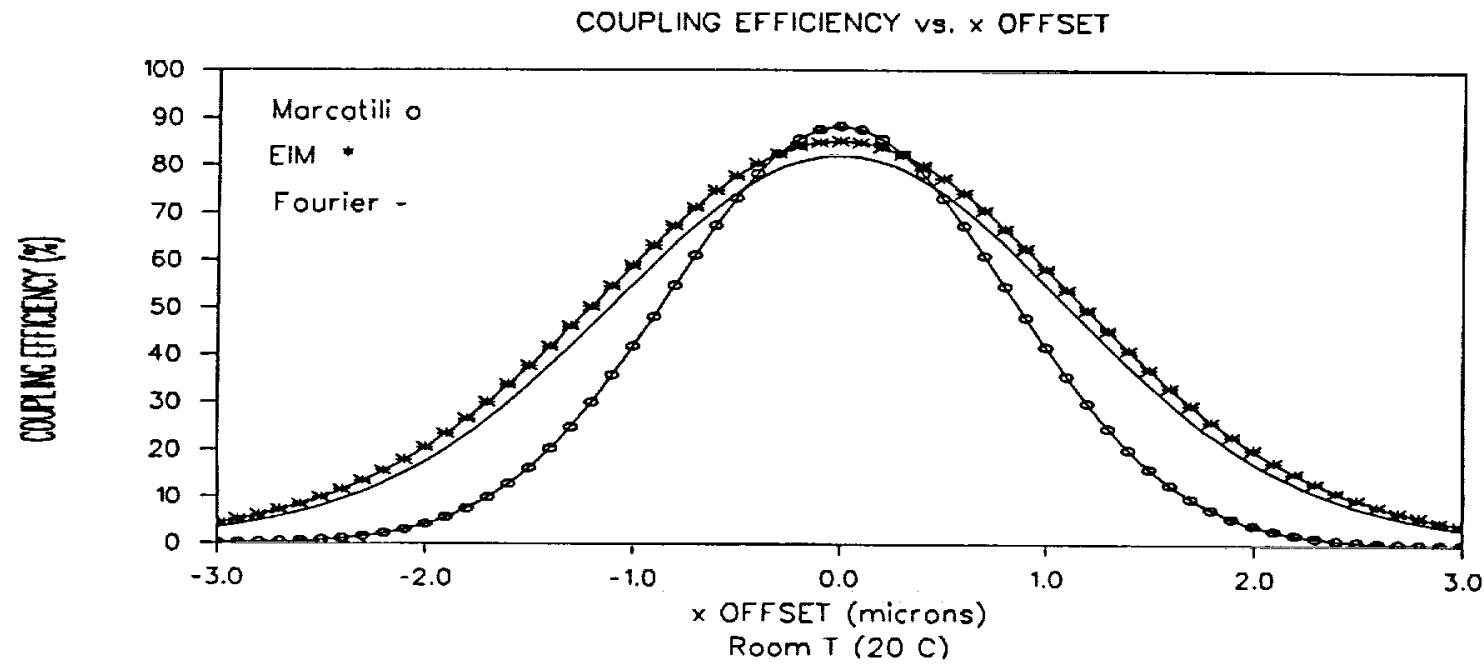

Figure 13: Calculated coupling efficiency vs. $x$ offset.

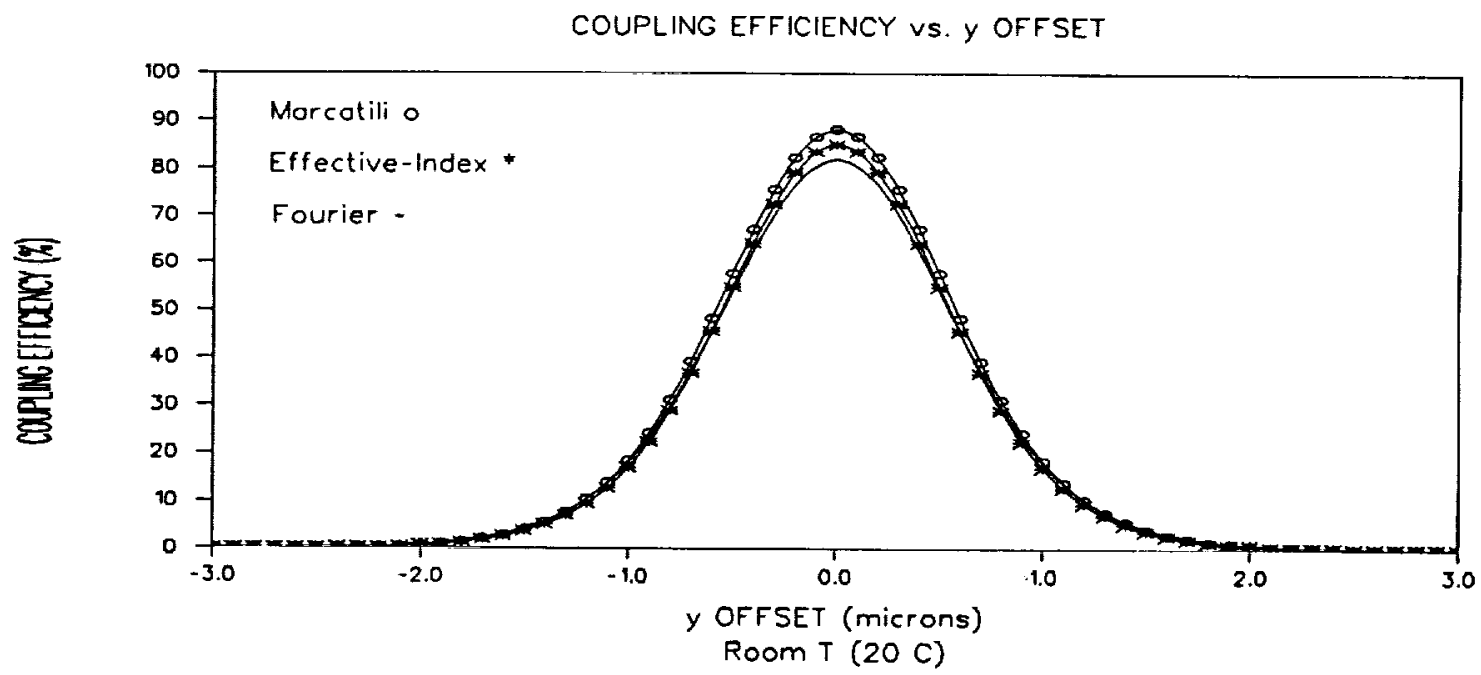

Figure 14: Calculated coupling efficiency vs. $y$ offset.

Similarly for the $y$ direction, keeping the $x$ alignment, the fiber position was varied from $-3 \mu m$ to $+3 \mu m$ from the position of alignment and the theoretical coupling efficiency versus $y$ offset is shown in Figure 14. The coupling efficiency versus $y$ offset results were nearly identical for the three methods. From the graphs in Figures 13 and 14 , 


\begin{tabular}{|c|c|c|}
\hline METHOD & MAXIMUM CE (RoOm T) & MAXIMUM CE $\left(300^{\circ} \mathrm{C}\right)$ \\
\hline EIM & $85.03 \%$ & $85.18 \%$ \\
\hline Marcatili & $88.16 \%$ & $88.15 \%$ \\
\hline Fourier & $81.99 \%$ & $81.98 \%$ \\
\hline
\end{tabular}

Table 3: Maximum Coupling Efficiency

it is apparent that alignment is more critical in the $y$ direction, since the rib field calculated is more tightly confined in the $y$ direction than the $x$.

The coupling efficiency results versus $x$ and $y$ offsets at $300^{\circ} \mathrm{C}$ were nearly identical to the room temperature results. The maximum coupling efficiency from the three techniques at both temperatures is given in tabular form in Table 3. From this theoretical study, it was determined that the predicted changes in the coupling efficiency with temperature are negligible and would be difficult to detect in practice.

\section{Summary}

Results indicate that the EIM was more accurate than Marcatili's approximation for both the fiber and rib waveguide. While results from the Fourier method were more accurate, the amount of time required to determine the coupling efficiency for $x$ and $y$ offsets was several orders of magnitude greater than that for the approximation methods.

These results indicate that the device is likely to perform properly in an environment with a temperature fluctuating between the two temperatures investigated. The calculated maximum coupling efficiency was higher than expected. Changes in the coupling efficiency with temperature were negligible because temperature induced variations in both the rib and fiber indices and dimensions were much less than expected.

The coupling efficiency can be increased by varying the rib waveguide geometry. By reducing the rib width to $2.4 \mu \mathrm{m}$ and the etched height outside the rib to $0.35 \mu \mathrm{m}$, the coupling efficiency increased to $88 \%$, using the Fourier method. This was $6 \%$ greater than that calculated using the present design. Hence, modeling is beneficial in the design process to improve the theoretical coupling efficiency.

\section{REFERENCES}

[1] Andrew Corporation, D-series elliptical fiber, 205170-820F-2.

[2] G.N. De Brabander, J.T. Boyd, G. Beheim, "Integrated Optical Ring Resonator With Micromechanical Diaphragm for Pressure Sensing," IEEE Photon. Tech. Lett., Vol. 6, No. 5, pp. 671-673, 1994.

[3] R.B. Dyott, J.R. Cozens, and D.G. Morris, "Preservation of Polarisation in Optical-Fibre Waveguides with Elliptical Cores," Electron. Lett., Vol. 15, No. 13, pp. 380-382, 1979.

[4] A. Kumar and R.K. Varshney, "Propagation Characteristics of Highly Elliptical Core Optical Waveguides: A Perturbation Approach," Opt. and Quant. Electron., Vol. 16, pp. 349-354, 1984.

[5] A. Kumar and R.K. Varshney, "Propagation Characteristics of Dual-Mode Elliptical-Core Optical Fibers," Opt. Lett., Vol. 14, No. 15, pp. 817-819, 1989.

[6] K.S. Chiang, "Geometrical Birefringence in a Class of Step-Index Fiber," J. Lightwave Tech., Vol. LT-5, No. 2, pp. $737-744,1987$. 
[7] R.M. Knox and P.P. Toulious, "Integrated Circuits for the Millimeter Through Optical Frequency Range," Proc. MRI Symposium on Submillimeter Waves, Polytechnic Press, Brooklyn, pp. 497-516, 1970.

[8] G.B. Hocker and W.K. Burns, "Mode Dispersion in Diffused Channel Waveguides by the Effective Index Method," Appl. Opt., Vol. 16, No. 1, pp. 113-118, 1977.

[9] D. Marcuse, Theory of Dielectric Optical Waveguides, pp. 7-8, 49-59, Academic Press, New York, 1991.

[10] V. Ramaswamy, "Strip-loaded film waveguide," Bell. Syst. Tech. J., Vol. 53, pp. 697-705, 1974.

[11] K.S. Chiang, "Performance of the Effective-Index Method," Opt. Lett., Vol. 16, No. 10, pp. 714-716, 1991.

[12] M.L. Tuma and G. Beheim, "Calculated Coupling Efficiency Between an Elliptical-Core Optical Fiber and a Silicon Oxynitride Rib Waveguide," Micro-Optics/Micromechanics and Laser Scanning and Shaping, SPIE, Vol. 2383, pp. 199-210, 1995.

[13] A. Kumar, D.F. Clark, and B. Culshaw, "Explanation of Errors Inherent in the Effective-Index Method for Analyzing Rectangular-Core Waveguides," Opt. Lett., Vol. 13, pp. 1129-1131, 1988.

[14] K.S. Chiang, "Analysis of Optical Fibers by the Effective-Index Method," Appl. Opt., Vol. 25, No. 3, pp. 348-354, 1986.

[15] E.A.J. Marcatili, "Dielectric Rectangular Waveguide and Directional Coupler for Integrated Optics," Bell. Syst. Tech. J., 48, pp. 2071-2102, 1969.

[16] A. Kumar, K. Thyagarajan, and A.K. Ghatak, "Analysis of Rectangular-Core Dielectric Waveguides: An Accurate Perturbation Approach," Opt. Lett., Vol. 8, No. 1, pp. 63-65, 1983.

[17] R.K. Varshney and A. Kumar, "A Simple and Accurate Modal Analysis of Strip-Loaded Optical Waveguides with Various Index Profiles," J. Lightwave Tech., Vol. 6, No. 4, pp. 601-606, 1988.

[18] D. Marcuse, "Solution of the Vector Wave Equation for General Dielectric Waveguides by the Galerkin Method," J. Quant. Electron., Vol. 28, No. 2, pp. 459-465, 1992.

[19] C.H. Henry and B.H. Verbeek, "Solution of the Scalar Wave Equation for Arbitrarily Shaped Dielectric Waveguides by Two-Dimensional Fourier Analysis," J. Lightwave Tech., Vol. 7, No. 2, pp. 308-313, 1989.

[20] A. Weisshaar and J. Li, Dept. of Electrical and Computer Engineering, Oregon State University, Oregon, FORTRAN programs written to determine propagation characteristics of optical waveguides.

[21] R.G. Hunsperger, A. Yariv, and A. Lee, "Parallel End-Butt Coupling for Optical Integrated Circuits," Appl. Opt., Vol. 16, No. 4, pp. 1026-1032, 1977.

[22] E.J. Murphy and T.C. Rice, "Self-Alignment Technique for Fiber Attachment to Guided Wave Devices," IEEE J. Quant. Electron., Vol. QE-22, No. 6, pp. 928-932, 1986.

[23] Y. Cai et. al., "An Effective Method for Coupling Single-Mode Fiber to Thin Film Waveguide," J. Lightwave Tech., Vol. 9, No. 5, pp. 577-583, 1991.

[24] D.J. Vezzetti and M. Munowitz, "Analysis of Finite Rib Waveguides by Matrix Methods," J. Lightwave Tech., Vol. 8, No. 6, pp. 889-893, 1990. [25] R.G. Hunsperger, Integrated Optics: Theory and Technology, 2nd ed., pp. 39-43, Springer-Verlag, New York,
1982 .

[26] M.J. Robertson, S. Ritchie, and P. Dayan, "Semiconductor Waveguides: Analysis of Coupling Between Rib Waveguides and Optical Fibres," Integrated Optical Circuit Engineering II, Proc. SPIE, Vol. 578, pp. 184-191,
1985. 


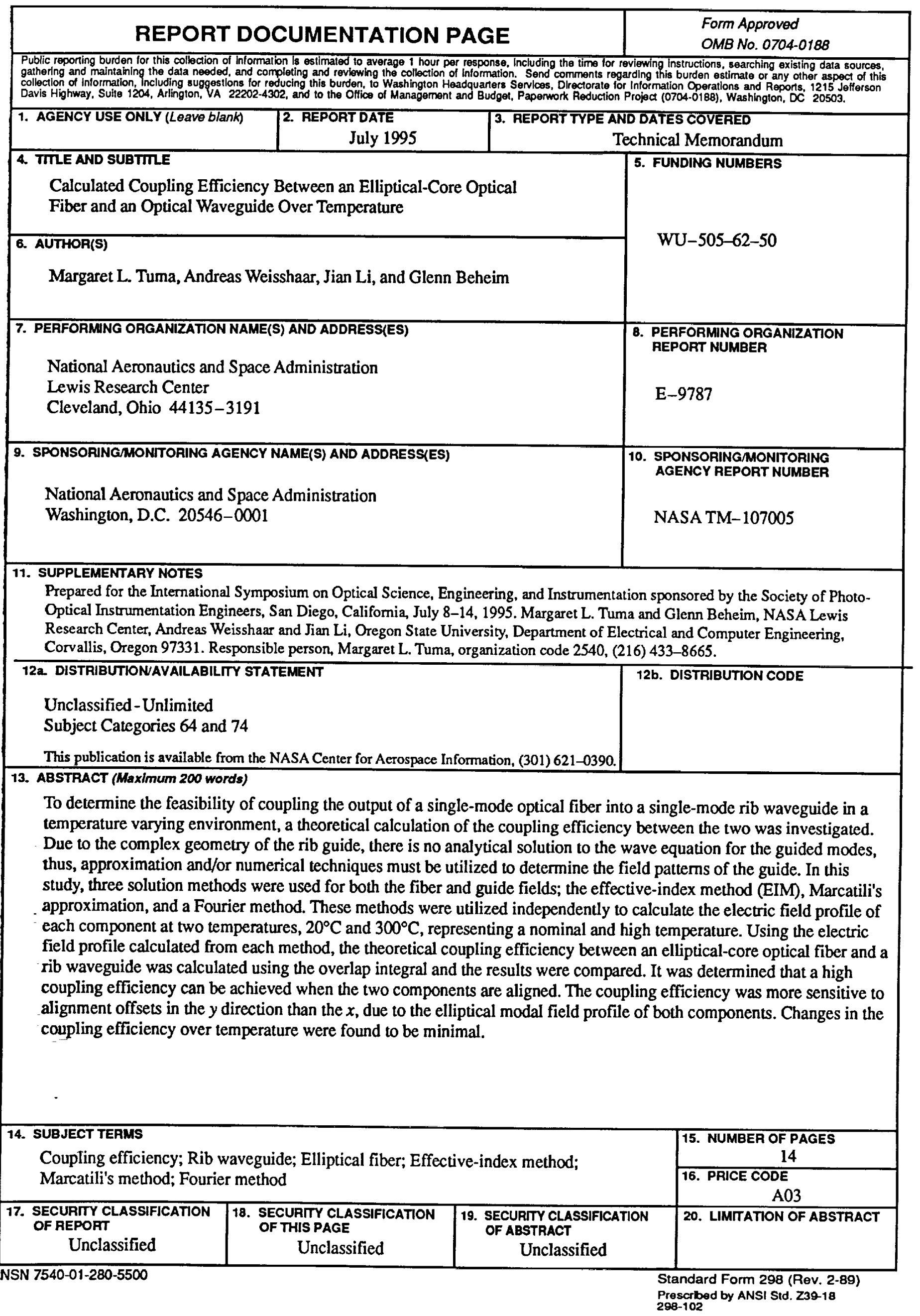


